\title{
Quantitative Assessment of Ambiguities in Plasmodium Propagation in Terms of Complex Networks and Rough Sets
}

\author{
Krzysztof Pancerz \\ Chair of Computer Science, Faculty of Mathematics and Natural Sciences \\ Prof. S. Pigonia Str. 1 \\ 35-310 Rzeszów, Poland \\ kpancerz@ur.edu.pl
}

\begin{abstract}
A Physarum machine is a biological computing device implemented in the plasmodium of Physarum polycephalum, a one-cell organism able to build large and manifold networks of protoplasmic veins for solving different computational tasks. In the paper, we propose to use complex networks as an underlying model of plasmodium propagation in Physarum machines. For such models, we define a measure, derived from rough set theory, for quantitative assessment of the cohesion of plasmodium connections between distinguished regions of interest. Rough sets are an appropriate tool to deal with some ambiguities which appear in plasmodium propagation.
\end{abstract}

\section{CCS Concepts}

-Computing methodologies $\rightarrow$ Modeling methodologies;

\section{Keywords}

Physarum machines; Plasmodium propagation; Complex networks; Rough sets

\section{INTRODUCTION}

There are a lot of systems composed by a large number of highly interconnected dynamical units. Biological and chemical systems, neural networks, social interacting species are the examples of them (see [2]). To model some properties of such systems, we can use graphs whose nodes represent the dynamical units, and whose links describe the interactions between them. In case of a large and manifold wiring architecture, the graphs are considered in terms of complex networks, i.e., networks whose structures are irregular, complex and dynamically evolving in time.

In the paper, we propose to use complex networks as an underlying model of plasmodium propagation in the so called Physarum machines. A Physarum machine [1] is a

Permission to make digital or hard copies of all or part of this work for personal or classroom use is granted without fee provided that copies are not made or distributed for profit or commercial advantage and that copies bear this notice and the full citation on the first page. To copy otherwise, to republish, to post on servers or to redistribute to lists, requires prior specific permission and/or a fee. BICT 2017, March 15-16, Hoboken, United States

ISBN 978-1-63190-148-5

DOI: 10.4108/eai.22-3-2017.152403

Copyright (๑) 2017 EAI biological computing device experimentally implemented in the plasmodium of Physarum polycephalum. The Physarum machine comprises an amorphous yellowish mass with networks of protoplasmic veins (see Figure 1), programmed by spatial configurations of attracting and/or repelling stimuli. The networks of protoplasmic veins show properties related to irregularity, complexity and dynamical evolution in time.

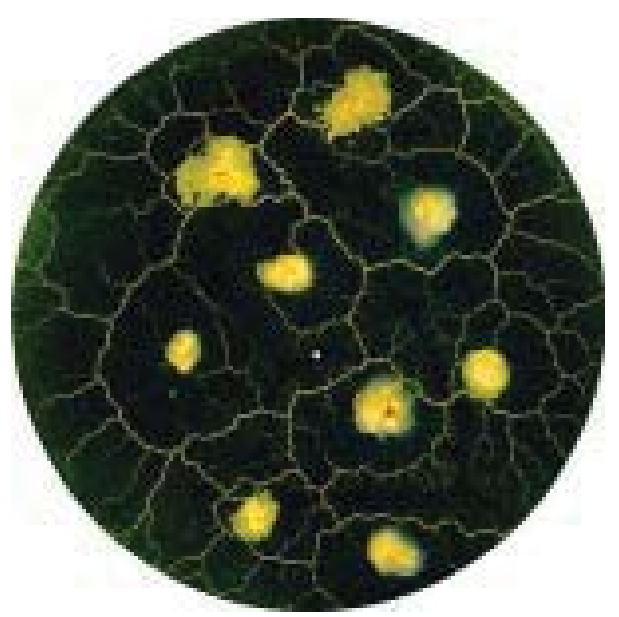

Figure 1: An amorphous yellowish mass with networks of protoplasmic veins.

In the literature, a variety of measures describing properties of complex networks has been proposed (see [2]). In the paper, we propose to use rough set theory for defining some measure characterizing ambiguities in plasmodium propagation in Physarum machines modeled by complex networks. This measure can be used for quantitative assessment of the cohesion of plasmodium connections between distinguished regions of interest.

The measure is defined analogously to the measure, proposed in [3], for assessment of the cohesion of saccade connections between object components in visual stimuli used in eye-tracking techniques. Rough sets theory proposed by Z . Pawlak is a mathematical tool to deal with rough (ambiguous, imprecise) concepts in the universe of discourse (cf. [6]). A rough set approach seems to be a suitable tool in case of behavior of Physarum machines whilst one can notice some ambiguities in plasmodium propagation (cf. [5], [8]). The 
presented approach refers to a general framework for the study of approximation using the notion of neighborhood systems proposed by T.Y. Lin (cf. [4]).

\section{RUDIMENTS OF ROUGH SETS}

The idea of rough sets proposed by Z. Pawlak (see [6]) consists of the approximation of a given set by a pair of sets, called the lower and the upper approximation of this set. In this section, we recall only necessary definitions, notions and notation concerning rough sets. For more detailed information, we refer the readers to [7] and [10].

Let $U \neq \emptyset$ be a finite set of objects we are interested in and $R$ be any binary relation over $U$. The image of $u \in U$ under $R$ is defined as $R(u)=\left\{u^{\prime} \in U:\left(u, u^{\prime}\right) \in R\right\}$. With each subset $X \subseteq U$ and any binary relation $R$ over $U$, we associate two subsets:

- $\underline{R}(X)=\{u \in U: R(u) \subseteq X\}$,

- $\bar{R}(X)=\{u \in U: R(u) \cap X \neq \emptyset\}$,

called the $R$-lower and $R$-upper approximation of $X$, respectively (cf. [9]). The $R$-lower approximation of $X$ consists of each element $u \in U$ whose image is wholly included in $X$. The $R$-upper approximation of $X$ consists of each element $u \in U$ whose image is partly included in $X$, i.e., each element $u \in U$ such that there exists at least one element of the image of $u$ that belongs to $X$. If $\underline{R}(X) \subset \bar{R}(X)$, then the set $X$ is rough, otherwise (i.e., $\underline{R}(X)=\bar{R}(X)$ ), the set $X$ is sharp.

Roughness of a set can be characterized numerically. To this end, the accuracy of approximation of $X$ with respect to $R$ is defined as:

$$
\alpha_{R}(X)=\frac{\operatorname{card}(\underline{R}(X))}{\operatorname{card}(\bar{R}(X))},
$$

where $\operatorname{card}$ denotes the cardinality of the set and $X \neq \emptyset$.

Some relaxed definition was proposed by W. Ziarko in the Variable Precision Rough Set Model (VPRSM) [10]. The standard set inclusion is replaced with the majority set inclusion in definitions of approximations. Let $0 \leq \beta<0.5$ and card denote the cardinality of the set. The $R_{\beta}$-lower approximation of $X$ is defined as:

$$
\underline{R}^{\beta}(X)=\{u \in U: R(u) \stackrel{\beta}{\subseteq} X\},
$$

where

$$
R(u) \stackrel{\beta}{\subseteq} X \text { if and only if } 1-\frac{\operatorname{card}(R(u) \cap X)}{\operatorname{card}(R(u))} \leq \beta .
$$

The $R$-lower approximation of $X$ consists of each element $u \in U$ whose image is mostly included in $X$, i.e., each element $u \in U$ such that a majority of elements of the image of $u$ belongs to $X$.

\section{PHYSARUM MACHINES}

In the Physarum machines, the plasmodium of Physarum polycephalum looks for attractants, propagates protoplasmic veins towards them, feeds on them and goes on. A network of protoplasmic veins, connecting the original points of plasmodium and those attractants, is formed. Each original point of plasmodium, and each attractant occupied by plasmodium, is called an active point in the Physarum machine.
Activated repellents can avoid or annihilate propagation of protoplasmic veins towards activated attractants.

Formally, a structure of the Physarum machine can be described as a triple $\mathcal{P} \mathcal{M}=(P h$, Attr, Rep) (cf. [5]), where:

- $P h=\left\{p h_{1}, p h_{2}, \ldots, p h_{k}\right\}$ is the set of original points of plasmodium,

- Attr $=\left\{a t t r_{1}, a t t t_{2}, \ldots, a t t r_{m}\right\}$ is the set of attractants;

- $R e p=\left\{r e p_{1}, r e p_{2}, \ldots, r e p_{n}\right\}$ is the set of repellents.

Let us consider a structure $\mathcal{P} \mathcal{M}=(P h$, Attr, Rep $)$ of the Physarum machine given in Figure 2. It is worth noting
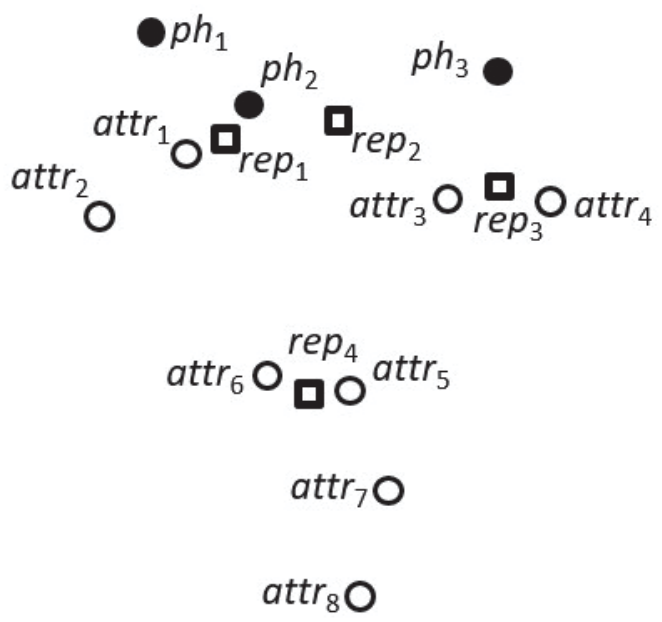

Figure 2: A structure $\mathcal{P} \mathcal{M}=(P h, A t t r, R e p)$ of the Physarum machine.

that, in the graphical presentation of structures of Physarum machines, we use the following symbols:

- filled circles corresponding to original points of plasmodium,

- empty circles corresponding to attractants,

- empty rectangles corresponding to repellents.

One can see that the components of the structure $\mathcal{P} \mathcal{M}=$ $(P h, A t t r, R e p)$ are as follows:

- $P h=\left\{p h_{1}, p h_{2}, p h_{3}\right\}$,

- $A t t r=\left\{a t t r_{1}, a t t_{2}, a t t_{3}, a t t_{4}, a t t r_{5}, a t t_{6}, a t t r_{7}, a t t r_{8}\right\}$,

- $R e p=\left\{r e p_{1}, r e p_{2}, r e p_{3}, r e p_{4}\right\}$.

Attractants and repellents can be activated/deactivated in time. Therefore, the network of protoplasmic veins changes in time. In general, a dynamics (behavior) of the Physarum machine $\mathcal{P} \mathcal{M}$ can be described by the family

$$
V=\left\{V^{t}\right\}_{t \in\left\{t_{0}, t_{1}, t_{2}, \ldots\right\}}
$$

of the sets of protoplasmic veins formed by plasmodium during its action and observed at discrete time instants $t_{0}, t_{1}, t_{2}$, $\ldots$, where $V^{t}=\left\{v_{1}^{t}, v_{2}^{t}, \ldots, v_{\text {card }\left(V^{t}\right)}^{t}\right\}$ is the set of all protoplasmic veins of plasmodium present at the time instant $t$ in $\mathcal{P} \mathcal{M}$. Each vein $v_{i}^{t} \in V^{t}$, where $i=1,2, \ldots, \operatorname{card}\left(V^{t}\right)$, is a pair $\left\langle\pi_{i s}^{t}, \pi_{i e}^{t}\right\rangle$ of active points in $\mathcal{P} \mathcal{M}$, i.e., $\pi_{i s}^{t} \in P h \cup A t t r$ and $\pi_{i^{e}}^{t} \in P h \cup$ Attr. 


\section{ASSESSMENT OF THE COHESION OF PLASMODIUM CONNECTIONS}

Formally, a complex network can be presented as a graph either undirected or directed. In the proposed approach, we consider complex networks represented by undirected graphs. It means that we are not interested in directions of edges.

An undirected graph $G=(N, E)$ consists of two sets $N$ and $E$ such that $N \neq \emptyset$ and $E$ is a set of unordered pairs of elements of $N$. The elements of $N=\left\{n_{1}, n_{2}, \ldots, n_{q}\right\}$ are the nodes of $G$, while the elements of $E=\left\{e_{1}, e_{2}, \ldots, e_{r}\right\}$ are the edges of $G$.

To build a model, in a form of a complex network, of behavior of the Physarum machine, we take into consideration a stable state of $\mathcal{P M}$, i.e., the state at a given time instant $t$, when the set of all protoplasmic veins formed by plasmodium is fixed, i.e., $V=\left\{v_{1}, v_{2}, \ldots, v_{\operatorname{card}(V)}\right\}$ (note that the superscript $t$ has been omitted).

Let $\mathcal{P} \mathcal{M}=(P h$, Attr, Rep $)$ be the Physarum machine and $G=(N, E)$ be a complex network modelling behavior of $\mathcal{P} \mathcal{M}$. The following bijective functions are used:

- $\nu: P h \cup A t t r \rightarrow N$ assigning a node to each original point of plasmodium as well as to each attractant,

- $\epsilon: V \rightarrow E$ assigning an edge to each protoplasmic vein.

In the set of nodes of the complex network, we can distinguish some regions of interest (ROIs), i.e., selected subsets of nodes identified for a particular purpose. Let $\Omega=$ $\left\{\omega_{1}, \omega_{2}, \ldots, \omega_{s}\right\}$ be a set of all regions of interest identified in the complex network. $\mathcal{N}=\left\{N_{\omega_{1}}, N_{\omega_{2}}, \ldots, N_{\omega_{s}}\right\}$ denotes a family of sets of nodes corresponding to regions of interest.

For each node $n \in N_{\omega_{1}} \cup N_{\omega_{2}} \cup \cdots \cup N_{\omega_{s}}$, we define its inter-region neighborhood:

$$
\begin{aligned}
& I R N(n)= \\
& =\left\{n^{\prime}:\left(n, n^{\prime}\right) \in E \text { and } \underset{\omega \in \Omega}{\exists}\left(n^{\prime} \in N_{\omega} \text { and } n \notin N_{\omega}\right)\right\} .
\end{aligned}
$$

On the basis of the complex network modeling behavior of a given Physarum machine $\mathcal{P} \mathcal{M}$, we define a measure, derived from rough set theory, for quantitative assessment of the cohesion of connections between regions of interest (cf. an analogous approach proposed in [3]).

Let $G=(N, E)$ be a complex network modeling behavior of a given Physarum machine $\mathcal{P} \mathcal{M}$ and $\omega_{i}, \omega_{j}$ be two distinguished regions of interest. The lower approximation $\operatorname{IRN}\left(\omega_{i} \rightarrow \omega_{j}\right)$ of the inter-region neighborhood, from $\omega_{i}$ to $\omega_{j}$, is defined as:

$$
\begin{aligned}
& \underline{\operatorname{IRN}}\left(\omega_{i} \rightarrow \omega_{j}\right)= \\
& =\left\{n \in N_{\omega_{i}}: \operatorname{IRN}(n) \neq \emptyset \text { and } \operatorname{IRN}(n) \subseteq N_{\omega_{j}}\right\} .
\end{aligned}
$$

The upper approximation $\overline{I R N}\left(\omega_{i} \rightarrow \omega_{j}\right)$ of the inter-region neighborhood, from $\omega_{i}$ to $\omega_{j}$, is defined as:

$$
\overline{I R N}\left(\omega_{i} \rightarrow \omega_{j}\right)=\left\{n \in N_{\omega_{i}}: \operatorname{IRN}(n) \cap N_{\omega_{j}} \neq \emptyset\right\} .
$$

The lower approximation $\underline{\operatorname{IRN}}\left(\omega_{i} \rightarrow \omega_{j}\right)$ of the interregion neighborhood consists of all nodes $N_{\omega_{i}}$ which are connected by inter-region edges with nodes from $N_{\omega_{j}}$ only. The upper approximation $\overline{I R N}\left(\omega_{i} \rightarrow \omega_{j}\right)$ of the inter-region neighborhood consists of all nodes $N_{\omega_{i}}$ which are connected at least by one inter-region edge with nodes from $N_{\omega_{j}}$.
The accuracy of approximation of the inter-region neighborhood can be defined analogously to the accuracy of approximation in rough set theory, i.e.:

$$
\alpha_{I R N}\left(\omega_{i} \rightarrow \omega_{j}\right)=\frac{\operatorname{card}\left(\underline{\operatorname{IRN}}\left(\omega_{i} \rightarrow \omega_{j}\right)\right)}{\operatorname{card}\left(\overline{\operatorname{IRN}}\left(\omega_{i} \rightarrow \omega_{j}\right)\right)} .
$$

We treat $\alpha_{I R N}\left(\omega_{i} \rightarrow \omega_{j}\right)$ as a measure of the cohesion of connections from the region of interest $\omega_{i}$ to the region of interest $\omega_{j}$. If $\alpha_{I R N}\left(\omega_{i} \rightarrow \omega_{j}\right)=1$, then the connection is the most coherent one.

In general, a measure of the cohesion of connections between regions of interest is not symmetrical, i.e.:

$$
\alpha_{I R N}\left(\omega_{i} \rightarrow \omega_{j}\right) \neq \alpha_{I R N}\left(\omega_{j} \rightarrow \omega_{i}\right),
$$

for $i, j=1,2, \ldots, s$ and $i \neq j$.

Let us consider a complex network (see Figure 3 ) modeling behavior of the Physarum machine $\mathcal{P} \mathcal{M}=(P h, A t t r, R e p)$ shown in Figure 2. The labels of edges have been omitted

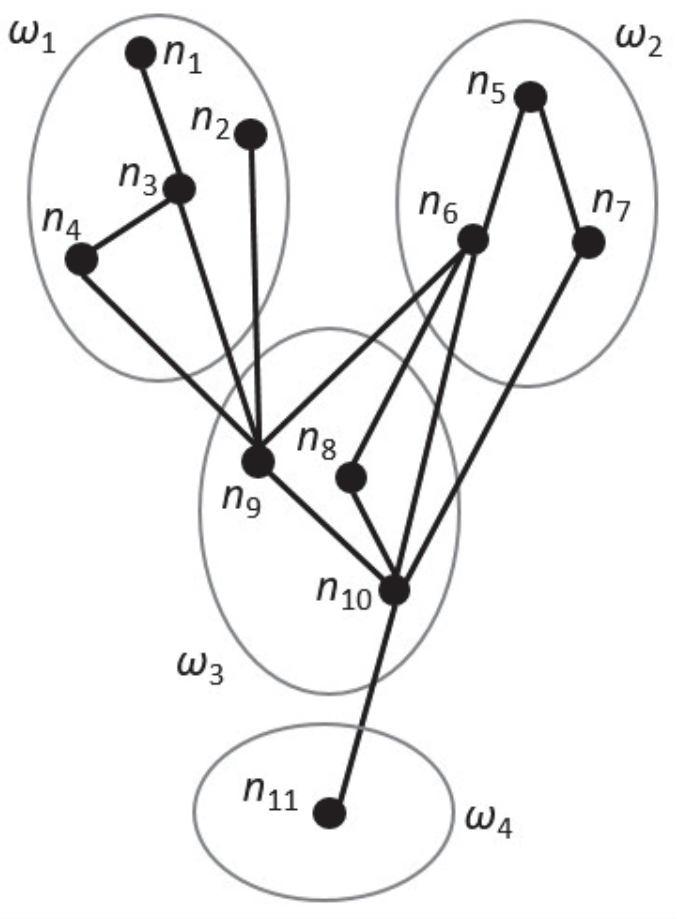

Figure 3: A fragment of a complex network modeling behavior of a given Physarum machine $\mathcal{P} \mathcal{M}$.

for better legibility. For instance, four regions of interest are identified with the following nodes:

- $N_{\omega_{1}}=\left\{n_{1}, n_{2}, n_{3}, n_{4}\right\}$,

- $N_{\omega_{2}}=\left\{n_{5}, n_{6}, n_{7}\right\}$,

- $N_{\omega_{3}}=\left\{n_{8}, n_{9}, n_{10}\right\}$

- $N_{\omega_{4}}=\left\{n_{11}\right\}$.

A measure of the cohesion of connections from the region of interest $\omega_{1}$ to the region of interest $\omega_{3}$ :

$$
\alpha_{I R N}\left(\omega_{1} \rightarrow \omega_{3}\right)=1
$$

because: 
- $\operatorname{IRN}\left(n_{1}\right)=\emptyset$,

- $\operatorname{IRN}\left(n_{2}\right)=\left\{n_{9}\right\} \subseteq N_{\omega_{3}}$,

- $\operatorname{IRN}\left(n_{3}\right)=\left\{n_{9}\right\} \subseteq N_{\omega_{3}}$,

- $\operatorname{IRN}\left(n_{4}\right)=\left\{n_{9}\right\} \subseteq N_{\omega_{3}}$,

and

- $\underline{\operatorname{IRN}}\left(\omega_{1} \rightarrow \omega_{3}\right)=\left\{n_{2}, n_{3}, n_{4}\right\}$,

- $\overline{\operatorname{IRN}}\left(\omega_{1} \rightarrow \omega_{3}\right)=\left\{n_{2}, n_{3}, n_{4}\right\}$.

A measure of the cohesion of connections from the region of interest $\omega_{3}$ to the region of interest $\omega_{1}$ :

$$
\alpha_{I R N}\left(\omega_{3} \rightarrow \omega_{1}\right)=0
$$

because:

- $\operatorname{IRN}\left(n_{8}\right)=\left\{n_{6}\right\} \nsubseteq N_{\omega_{1}}, \operatorname{IRN}\left(n_{8}\right) \cap N_{\omega_{1}}=\emptyset$,

- $\operatorname{IRN}\left(n_{9}\right)=\left\{n_{2}, n_{3}, n_{4}, n_{6}\right\} \nsubseteq N_{\omega_{1}}$, but $\operatorname{IRN}\left(n_{9}\right) \cap$ $N_{\omega_{1}}=\left\{n_{2}, n_{3}, n_{4}\right\}$

- $\operatorname{IRN}\left(n_{10}\right)=\left\{n_{6}, n_{7}, n_{11}\right\} \nsubseteq N_{\omega_{1}}, I R N\left(n_{10}\right) \cap N_{\omega_{1}}=\emptyset$

and

- $\underline{\operatorname{IRN}}\left(\omega_{3} \rightarrow \omega_{1}\right)=\emptyset$,

- $\overline{I R N}\left(\omega_{1} \rightarrow \omega_{3}\right)=\left\{n_{9}\right\}$.

One can see that $\alpha_{I R N}\left(\omega_{1} \rightarrow \omega_{3}\right) \neq \alpha_{I R N}\left(\omega_{3} \rightarrow \omega_{1}\right)$.

We slightly modify the definition of the lower and upper approximation of the inter-region neighborhood in case of the Variable Precision Rough Set Model (VPRSM).

Let $G=(N, E)$ be a complex network modeling behavior of a given Physarum machine $\mathcal{P} \mathcal{M}, \omega_{i}, \omega_{j}$ be two distinguished regions of interest, and $0 \leq \beta<0.5$. The $\beta$-lower approximation $\underline{\operatorname{IRN}}\left(\omega_{i} \rightarrow \omega_{j}\right)$ of the inter-region neighborhood, from $\omega_{i}$ to $\omega_{j}$, is given by:

$$
\begin{aligned}
& \underline{\operatorname{IRN}}^{\beta}\left(\omega_{i} \rightarrow \omega_{j}\right)= \\
& =\left\{n \in N_{\omega_{i}}: \operatorname{IRN}(n) \neq \emptyset \text { and } \operatorname{IRN}(n) \stackrel{\beta}{\subseteq} N_{\omega_{j}}\right\} .
\end{aligned}
$$

Now, one can see that the $\beta$-lower approximation of the inter-region neighborhood $\underline{\operatorname{IRN}}\left(\omega_{i} \rightarrow \omega_{j}\right)$ consists of all nodes $N_{\omega_{i}}$ which are connected by inter-region edges, in most cases (i.e., in terms of the majority set inclusion), with nodes from $N_{\omega_{j}}$.

A relaxed measure of the cohesion of connections from the region of interest $\omega_{i}$ to the region of interest $\omega_{j}$ has the form:

$$
\alpha_{I R N}^{\beta}\left(\omega_{i} \rightarrow \omega_{j}\right)=\frac{\operatorname{card}\left(\underline{\operatorname{IRN}}^{\beta}\left(\omega_{i} \rightarrow \omega_{j}\right)\right)}{\operatorname{card}\left(\overline{\operatorname{IRN}^{\beta}}\left(\omega_{i} \rightarrow \omega_{j}\right)\right)}
$$

for $0 \leq \beta<0.5$.

Let us consider a complex network, modeling behavior of a given Physarum machine $\mathcal{P} \mathcal{M}$, shown in Figure 3. A measure of the cohesion of connections from the region of interest $\omega_{3}$ to the region of interest $\omega_{2}$ :

$$
\alpha_{I R N}\left(\omega_{3} \rightarrow \omega_{2}\right)=\frac{1}{3}
$$

because:

- $\underline{\operatorname{IRN}}\left(\omega_{3} \rightarrow \omega_{2}\right)=\left\{n_{8}\right\}$,

- $\overline{I R N}\left(\omega_{3} \rightarrow \omega_{2}\right)=\left\{n_{8}, n_{9}, n_{10}\right\}$.
Let $\beta=0.5$, a relaxed measure of the cohesion of connections from the region of interest $\omega_{3}$ to the region of interest $\omega_{2}$ :

$$
\alpha_{I R N}^{0.5}\left(\omega_{3} \rightarrow \omega_{2}\right)=\frac{2}{3}
$$

because:

- $\underline{\operatorname{IRN}}^{0.5}\left(\omega_{3} \rightarrow \omega_{2}\right)=\left\{n_{8}, n_{10}\right\}$,

- $\overline{\operatorname{IRN}}^{0.5}\left(\omega_{2} \rightarrow \omega_{2}\right)=\left\{n_{8}, n_{9}, n_{10}\right\}$.

\section{CONCLUSIONS}

We have proposed a measure for quantitative assessment of the cohesion of plasmodium connections between distinguished regions of interest based on rough set theory. The proposed measure reflects some ambiguities which appear in plasmodium propagation. We can distinguish two main directions of the further research. The first one will concern comparing the proposed measure with classic measures, based on graph theory, describing properties of complex networks. The second one will concern extending the spectrum of measures by applying various rough set approaches.

\section{REFERENCES}

[1] A. Adamatzky. Physarum Machines: Computers from Slime Mould. World Scientific, 2010.

[2] S. Boccaletti, V. Latora, Y. Moreno, M. Chavez, and D.-U. Hwang. Complex networks: Structure and dynamics. Physics Reports, 424(4-5):175-308, 2006.

[3] B. Jaskuła, J. Szkoła, K. Pancerz, and A. Derkacz. Eye-tracking data, complex networks and rough sets: An attempt toward combining them. In J. Suzuki, T. Nakano, and H. Hess, editors, Proceedings of the 9th International Conference on Bio-inspired Information and Communications Technologies (BICT'2015), pages 167-173, New York City, New York, USA, 2015.

[4] T. Y. Lin. Topological and fuzzy rough sets. In R. Słowiński, editor, Intelligent Decision Support: Handbook of Applications and Advances of the Rough Sets Theory, pages 287-304. Springer Netherlands, Dordrecht, 1992.

[5] K. Pancerz and A. Schumann. Rough set models of Physarum machines. International Journal of General Systems, 44(3):314-325, 2015.

[6] Z. Pawlak. Rough Sets. Theoretical Aspects of Reasoning about Data. Kluwer Academic Publishers, Dordrecht, 1991.

[7] Z. Pawlak and A. Skowron. Rudiments of rough sets. Information Sciences, 177:3-27, 2007.

[8] A. Schumann and K. Pancerz. Roughness in timed transition systems modeling propagation of plasmodium. In D. Ciucci, G. Wang, S. Mitra, and W.-Z. Wu, editors, Rough Sets and Knowledge Technology, volume 9436 of Lecture Notes in Artificial Intelligence, pages 482-491. Springer International Publishing, 2015.

[9] Y. Yao and T. Lin. Generalization of rough sets using modal logics. Intelligent Automation and Soft Computing, 2(2):103-120, 1996.

[10] W. Ziarko. Variable precision rough set model. Journal of Computer and System Sciences, 46(1):39-59, 1993. 